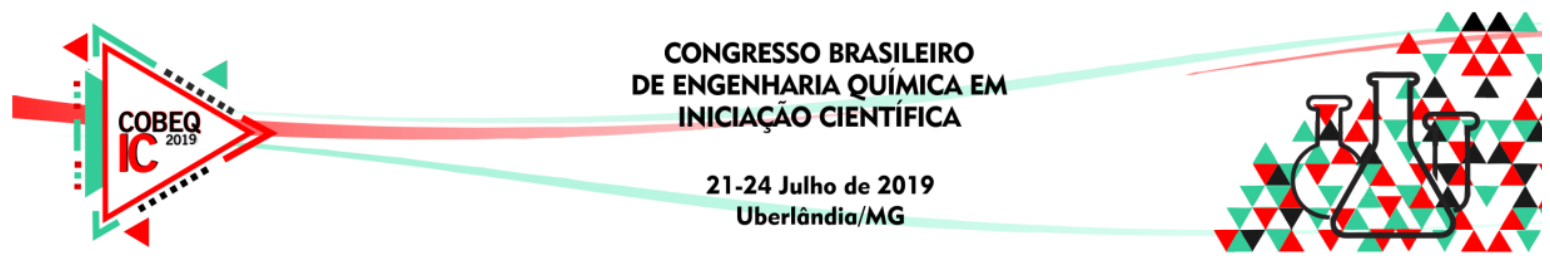

\title{
CARACTERIZAÇÃO FÍSICO-QUÍMICA DE SABONETES LÍQUIDOS E EM BARRA
}

\author{
A. D. MONTEIRO ${ }^{1}$ e V. M. SANTOS ${ }^{2}$ \\ ${ }^{1}$ Instituto Federal de Educação, Ciência e Tecnologia da Bahia \\ ${ }^{2}$ Instituto Federal de Educação, Ciência e Tecnologia da Bahia, Departamento de Engenharia \\ Química \\ E-mail para contato: amanda_monteiro93@hotmail.com; vanessa@ifba.edu.br
}

RESUMO - A caracterização de produtos de higiene pessoal permite conhecer o produto e obter informações possibilitando soluções e inovações. Assim, neste trabalho estudou-se sabonetes líquido e em barra através da determinação do $\mathrm{pH}$ e da viscosidade. $\mathrm{O} \mathrm{pH}$ foi medido através de metodologias padronizadas e outras propostas neste trabalho, enquanto a viscosidade de alguns sabonetes líquidos foi determinada através de viscosímetro rotacional. A partir da metodologia empregada, verificou-se que os sabonetes em barra, exceto aqueles de origem sintética, apresentaram $\mathrm{pH}$ básico. Essa alteração no $\mathrm{pH}$ pode danificar a barreira antibacteriana da pele, provocar ressecamento e irritações. Isso ocorre devido à diferença com o $\mathrm{pH}$ da pele cujo valor varia entre 4 e 6 , funcionando como uma barreira contra compostos químicos agressivos e microrganismos patogênicos. De forma geral, notou-se que os sabonetes líquidos apresentaram viscosidade dentro do valor mínimo determinado pela Anvisa e que alguns dos sabonetes apresentaram comportamento de fluidos newtonianos e os demais de nãonewtonianos pseudoplásticos. Adicionalmente, notou-se que as características dos produtos podem ser relacionadas à composição declarada no rótulo e que a viscosidade é estabelecida conforme a forma de aplicação e região do corpo na qual será usado. Este trabalho foi motivado como uma estratégia de aplicar conceitos da engenharia de produtos a fim de despertar interesse pela área de desenvolvimento de produtos e processos.

\section{INTRODUÇÃO}

A caracterização de produtos de higiene pessoal, bem como a obtenção de informações sobre tendências em formulações, processos e atributos de marketing permitem conhecer o produto e o mercado atual, a fim de propor alternativas em termos de matérias-primas e processos, alinhadas às demandas do consumidor em termos de qualidade, percepção e preocupação ambiental.

Dentre os produtos pertencentes à categoria de higiene pessoal, podem-se destacar os sabonetes. Estes são obtidos tradicionalmente por meio de óleos e gorduras vegetais e animais, que são substâncias constituídas preponderantemente por triacilgliceróis ou triglicerídeos [3(RCOO- $\left.\mathrm{CH}_{2}\right)$ ]. Esses compostos são formados a partir de ácidos carboxílicos de cadeia longa denominados ácidos graxos. Para produção do sabão, os triacilgliceróis sofrem uma reação de saponificação através da adição de uma base, geralmente $\mathrm{NaOH}$, produzindo glicerina $\left[3\left(\mathrm{CH}_{2} \mathrm{OH}\right)\right]$ e sais alcalinos dos ácidos graxos [3(R-COO$\left.\left.{ }^{-} \mathrm{Na}^{+}\right)\right](\mathrm{Castro}, 2009)$. 


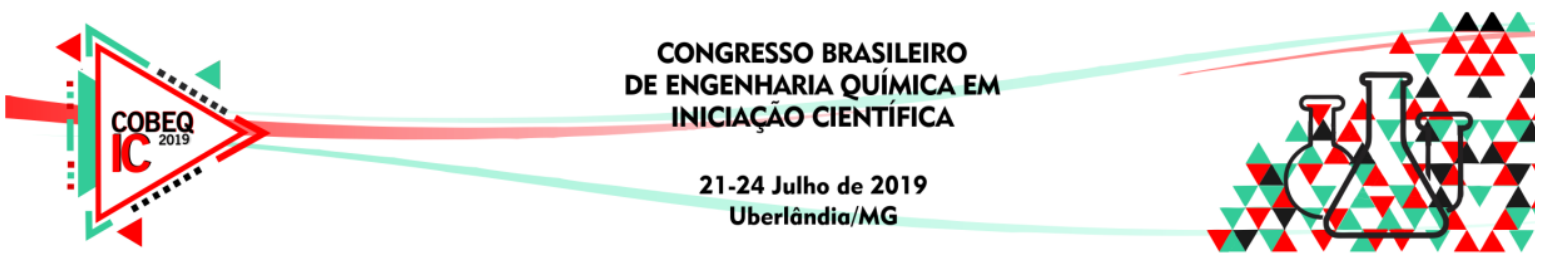

O sabonete comum é um sal oriundo de uma base forte e um ácido fraco, por isso hidrolisa quando em solução e o grupo hidroxila liberado é o responsável pelo $\mathrm{pH}$ alcalino característico desses produtos (Mendes et. al., 2016). Além dos sabonetes comuns, há os detergentes sintéticos (syndets), constituídos principalmente por tensoativos de origem sintética.

$\mathrm{O}$ pH é uma das propriedades mais importantes desse tipo de produto, visto que, pode influenciar na manutenção da saúde da pele. Este órgão é composto de suor e sebo, que representam uma manta de proteção natural contra compostos químicos agressivos e microrganismos patogênicos, apresentando pH entre 4 e 6 (Isenmann, 2015).

As características reológicas destes produtos são também propriedades relevantes, pois, definem aspectos relativos ao dimensionamento de equipamentos, processo de fabricação, forma de aplicação e qualidade dos produtos finais. Dentre essas propriedades, a viscosidade é importante do ponto de vista prático, no aspecto sensorial e na condução do desenvolvimento do produto e processo de fabricação (Flow, 2017).

Além dessas, características como cor, odor, sabor, sensação ao tato são propriedades determinantes para a aceitação de um produto pelo consumidor. Essas características são avaliadas por meio de ensaios organolépticos. Segundo descrição da ANVISA (2008) "ensaios organolépticos são procedimentos utilizados para avaliar as características de um produto, detectáveis pelos órgãos dos sentidos: aspecto, cor, odor, sabor e tato".

\section{OBJETIVOS GERAIS E ESPECÍFICOS}

O objetivo geral deste trabalho foi caracterizar sabonetes líquido e em barra. Para alcançar o objetivo geral, os seguintes objetivos específicos foram buscados: estabelecer os produtos comerciais a serem estudados, caracterizar os produtos através de propriedades físico-químicas, relacionar as propriedades físicas e químicas do produto à sua composição declarada nos rótulos e relacionar a composição às tendências e expectativas do mercado.

\section{METODOLOGIA}

Para a realização do trabalho selecionou-se sabonetes em barra e líquidos, considerandose a alta penetração destes produtos no mercado brasileiro, maior disponibilidade e custo acessível. O critério foi selecionar produtos com diferentes atributos ou apelos de marketing e cujas composições pudessem resultar em produtos com propriedades físico-químicas diferentes, por exemplo, $\mathrm{pH}$ diferentes. Assim, os sabonetes em barra adquiridos no mercado foram divididos em diferentes grupos: convencional com sebo, convencional com base vegetal, base sintética, sabonetes glicerinados que podem pertencer a um dos três grupos mencionados anteriormente, desodorante e antimicrobiano. Para os sabonetes líquidos, foram selecionadas marcas disponíveis no mercado com diferentes atributos ou apelos de marketing.

\subsection{Determinação do pH e da Viscosidade}

A análise físico-química para determinação do pH dos sabonetes foi realizada conforme Guia de Controle de Qualidade de Produtos Cosméticos disponibilizado pela ANVISA (2008), sendo as medidas realizadas em triplicata. 


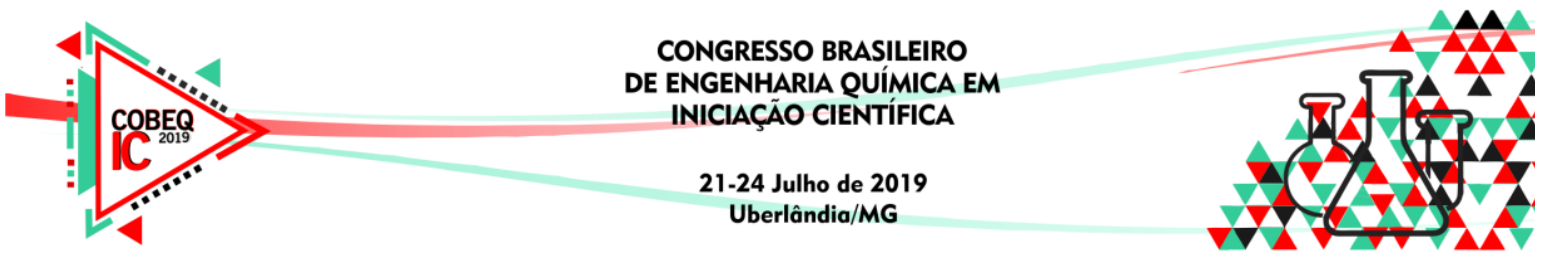

Sabonete em barra: Para medição do $\mathrm{pH}$ dos sabonetes em barra, pesou-se $10 \mathrm{~g} \pm 0,01 \mathrm{de}$ aparas dos sabonetes previamente cortados ao meio e raspados na superfície interna com o auxílio de uma faca. Tais aparas foram dissolvidas em $100 \mathrm{~mL}$ de água destilada, obtendo-se soluções posteriormente armazenadas em frascos plásticos fechados. As soluções preparadas foram agitadas novamente após 2 horas com o objetivo de acelerar o processo de dissolução.

A primeira medida de $\mathrm{pH}$ foi executada 2 horas após o preparo das soluções. Após 24 horas, mais duas medições foram realizadas em equipamentos distintos. Após 48 horas, foi realizada uma diluição adicional com a adição de $50 \mathrm{~mL}$ de água destilada, resultando em soluções a $6,7 \%$, que ficaram em repouso por dois dias até a nova medição. Tal diluição foi feita com o objetivo de avaliar a possibilidade de se trabalhar com uma solução menos concentrada e, portanto, com maior facilidade de dissolução, sendo uma variação da metodologia original.

Sabonete líquido: Para medição do pH dos sabonetes líquidos, imergiu-se o eletrodo do pHmetro em aproximadamente $30 \mathrm{~mL}$ de cada sabonete líquido em um béquer. Adicionalmente, foi realizada uma diluição com 2,5 g do sabonete líquido em $25 \mathrm{~mL}$ de água destilada, resultando em soluções a $10 \% \mathrm{~m} / \mathrm{v}$. Tal diluição foi feita com o objetivo de avaliar a possibilidade de se trabalhar com menor quantidade de produto.

Viscosidade: A viscosidade das amostras foi determinada através do viscosímetro da marca Fungilab, modelo visco basic plus, com spindles $\mathrm{L}_{2}$, $\mathrm{L}_{3}$ e $\mathrm{L}_{4}$ com diferentes rotações para verificar quais condições melhor se adequavam à realização da medida e como tal propriedade comportava-se a essas variações. Inicialmente, nivelou-se o aparelho, o spindle foi mergulhado na amostra até a marca (sulco) da haste do fuso (spindle) e efetuou-se a leitura após aproximadamente um minuto do acionamento do viscosímetro. Para sabonetes líquidos recomenda-se que a viscosidade seja pelo menos $2000 \mathrm{cp}$ (Nardin, 2013).

\subsection{Compreensão dos Rótulos dos Produtos e Avaliação das Características Organolépticas}

Nesta etapa, identificou-se os componentes presentes na composição por meio da leitura dos rótulos. A função de cada componente foi identificada com base na literatura. $\mathrm{O}$ conhecimento da função desses componentes permitiu correlacionar a composição com as características organolépticas, viscosidade e diferença de $\mathrm{pH}$ de cada produto.

As amostras foram classificadas também de acordo com as seguintes características organolépticas: cor, odor típico, opacidade e translucidez.

\section{RESULTADOS E DISCUSSÃO}

A partir da metodologia empregada, determinou-se a viscosidade de quatro dos sabonetes líquidos, cujos valores médios obtidos estão apresentados na Tabela 1. Alguns dos sabonetes apresentaram grande diferença na viscosidade ao modificar-se o spindle. Por isso, a medição foi adequada a partir da variação da velocidade de rotação, baseando-se no manual do viscosímetro, o qual indica que para a obtenção de valores adequados, a leitura deveria estar compreendida entre 15 e $95 \%$ do fundo de escala. 


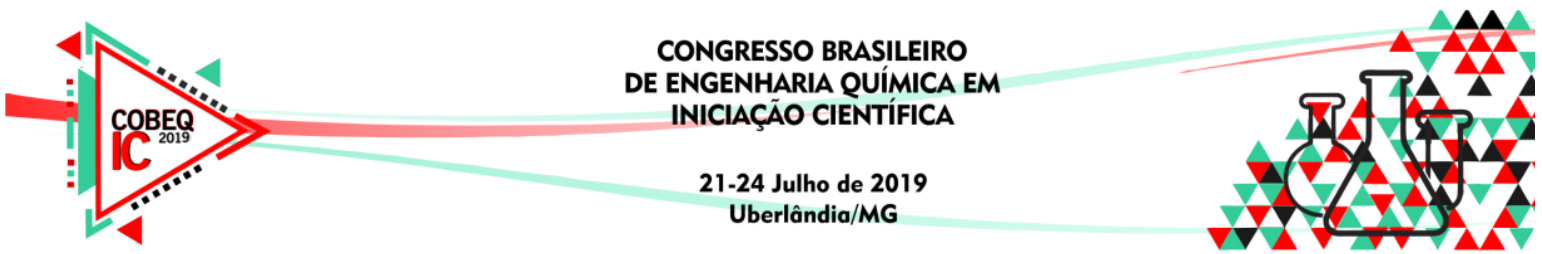

Desta forma, para o sabonete codificado por LX, as velocidades de 10 e $12 \mathrm{rpm}$ forneceram medidas mais adequadas e, para o sabonete codificado por JB, a velocidade de 20 rpm foi a mais apropriada utilizando-se o spindle $\mathrm{L}_{3}$. Enquanto para o sabonete $\mathrm{BF}$, o spindle $\mathrm{L}_{2}$ permitiu a obtenção da viscosidade em diversas velocidades e o spindle $\mathrm{L}_{4}$ foi o mais apropriado à medição do sabonete NS por permitir a realização de medidas em um maior intervalo de velocidade.

Tabela 1 - Medidas de viscosidade das diferentes marcas de sabonete líquido

\begin{tabular}{|c|c|c|c|}
\hline \multirow{2}{*}{ Marca } & \multicolumn{3}{|c|}{ Spindle } \\
\cline { 2 - 4 } & $\mathbf{L}_{\mathbf{2}}$ & $\mathbf{L}_{\mathbf{3}}$ & $\mathbf{L}_{\mathbf{4}}$ \\
\hline $\mathbf{L X}$ & - & 6097,8 & - \\
\hline JB & Sem leitura & 2866,98 & $3652,1 \pm 15,0$ \\
\hline $\mathbf{B F}$ & 1357,5 & 1006,5 & $2000 \pm 50$ \\
\hline NS & Sem leitura & 6572,1 & 6198,7 \\
\hline
\end{tabular}

De modo geral, notou-se que quanto menor a viscosidade maior o diâmetro do spindle a ser empregado, para que haja o aumento da superfície de contato entre fluido e cilindro provocando um maior torque. Esse comportamento é atribuído ao fato de que fluidos com viscosidade menor necessitam de uma área com maior cisalhamento, a fim de obter um torque mínimo de $20 \%$ e vice-versa para fluidos com maior viscosidade (Felippe et. al., 20-).

Alguns dos sabonetes cuja viscosidade foi determinada apresentaram características de fluidos newtonianos (LX e JB), enquanto os demais produtos comportaram-se como nãonewtonianos pseudoplásticos (BF e NS). Adicionalmente, verificou-se que os diferentes valores de viscosidade e características reológicas se relacionam à região e modo de uso dos sabonetes. Sendo assim, destaca-se a menor viscosidade do sabonete JB, visto que tal produto deve apresentar menor valor desta propriedade para facilitar a aplicação, considerando-se que este é destinado a utilização em bebês sendo aplicado possivelmente com uma única mão.

Conforme metodologia apresentada, determinou-se também o $\mathrm{pH}$ das diversas marcas de sabonetes. Desta forma, obteve-se a Tabela 2 que apresenta as informações comerciais dos sabonetes em barra utilizados em função do grupo escolhido. Além disso, obteve-se as Tabelas 3 e 4 que mostram os resultados das análises de $\mathrm{pH}$, para os sabonetes em barra e líquidos, respectivamente. A partir dos resultados obtidos, notou-se que a diluição adicional avaliada não trouxe alteração ao $\mathrm{pH}$ podendo, portanto, ser utilizada nos próximos ensaios.

Tabela 2 - Amostras dos sabonetes em barra

\begin{tabular}{|c|c|c|c|}
\hline Categoria & Marca/Fabricante & Categoria & Marca/Fabricante \\
\hline Antibacteriano & RAF, PRX e LFB & Convencional com sebo & NPL, ES e JB \\
\hline Desodorante & RCF & Base vegetal & $\begin{array}{c}\text { GB, PH, SCU, NMB, } \\
\text { NE, TBS, PMV e SL }\end{array}$ \\
\hline Glicerinado & RCF, GB, PH e TBS & Sintético & DV \\
\hline
\end{tabular}

De acordo com a avaliação dos resultados apresentados nas Tabelas 3 e 4 , notou-se que os sabonetes em barra das diversas categorias, que podem ser oriundos de ácidos graxos de origem animal ou vegetal apresentaram $\mathrm{pH}>9,0$, inclusive os sabonetes destinados ao uso em bebês, glicerinados e de coco. Nenhum sabonete ultrapassou o valor de $\mathrm{pH}$ máximo de 11,5 determinado pela Anvisa o que alteraria a classificação do produto para Grau de Risco 2. 


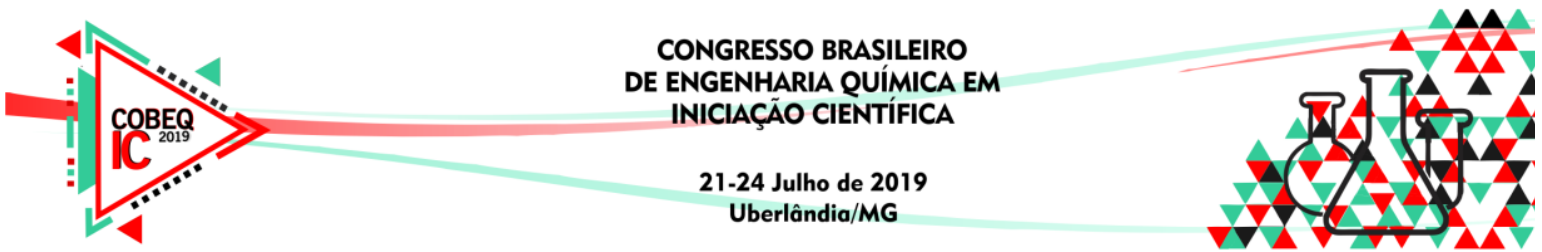

Os sabonetes em barra da marca DV e os líquidos apresentaram pH mais próximo ao fisiológico. Estes apresentam detergentes sintéticos na formulação, que atuam da mesma forma em termos de limpeza que os sabonetes convencionais. No entanto, os sintéticos, em sua maioria, são sais de ácidos sulfônicos oriundos de ácidos e base forte, portanto não sofrem hidrólise e mantêm o pH neutro, diferentemente dos sabonetes convencionais que são sais oriundos de um ácido fraco e base forte e hidrolisam quando em solução liberando hidroxila, responsável pela elevação do $\mathrm{pH}$ da pele.

Estes por apresentarem valores de $\mathrm{pH}$ muito básicos podem destruir o manto ácido da pele, que atua como uma barreira antibacteriana, desestruturar as lamelas da epiderme, contribuir para o ressecamento devido a maior perda transepidérmica de água e favorecer a entrada de potenciais irritantes e alérgenos. Assim, os sabonetes convencionais, dependendo da resposta de cada usuário, podem ser agressivos, principalmente para a pele infantil e de idosos, que podem requerer um maior tempo para recuperar o $\mathrm{pH}$ ácido após a alteração provocada pelo uso do sabonete.

Tabela 3 - Medidas de $\mathrm{pH}$ obtidas para diferentes marcas de sabonetes em barra

\begin{tabular}{|c|c|c|c|c|c|}
\hline Amostra & $\mathbf{p H}$ & $\begin{array}{c}\text { Desvio } \\
\text { padrão }\end{array}$ & Amostra & $\mathbf{p H}$ & $\begin{array}{c}\text { Desvio } \\
\text { padrão }\end{array}$ \\
\hline RAF & 10,17 & 0,18 & LFB & 10,45 & 0,12 \\
\hline PRX & 9,97 & 0,30 & PMV & 10,21 & 0,12 \\
\hline RCF & 10,48 & 0,51 & SCU & 10,29 & 0,21 \\
\hline NPL & 10,61 & 0,39 & SL & 10,90 & 0,26 \\
\hline GB & 10,42 & 0,44 & JB & 11,06 & 0,02 \\
\hline PH & 10,50 & 0,42 & NMB & 10,57 & 0,05 \\
\hline DV & 7,11 & 0,23 & NE & 9,90 & 0,02 \\
\hline ES & 10,33 & 0,37 & TBS & 10,11 & 0,06 \\
\hline
\end{tabular}

Tabela 4 - Medidas de pH obtidas para diferentes marcas de sabonetes líquidos

\begin{tabular}{|c|c|c|}
\hline Amostra & pH médio & Desvio padrão \\
\hline NS & 6,35 & 0,31 \\
\hline LX & 5,32 & 0,24 \\
\hline BF & 4,82 & 0,12 \\
\hline JB & 4,53 & 0,02 \\
\hline DTL & 4,39 & 0,13 \\
\hline NH & 5,85 & 0,09 \\
\hline NCS & 4,65 & 0,04 \\
\hline
\end{tabular}

De acordo com a análise da composição dos produtos, verificou-se que os sabonetes em barra, com exceção do sabonete sintético, apresentam ingredientes provenientes de ácidos graxos de origem animal ou vegetal. Enquanto para os sabonetes líquidos, verificou-se a presença de tensoativos sintéticos e de matérias-primas responsáveis pelo ajuste de viscosidade. Notou-se também nas formulações a existência de conservantes que são utilizados para impedir o desenvolvimento de microrganismos. Esses compostos são alvo de controvérsias pelo potencial irritativo e alergênico, levando ao surgimento de apelos de marketing como "naturais (green)" ou "livre de conservantes (preservative-free)". Com relação às propriedades organolépticas observou-se que a cor, odor e características de opacidade e translucidez dos 


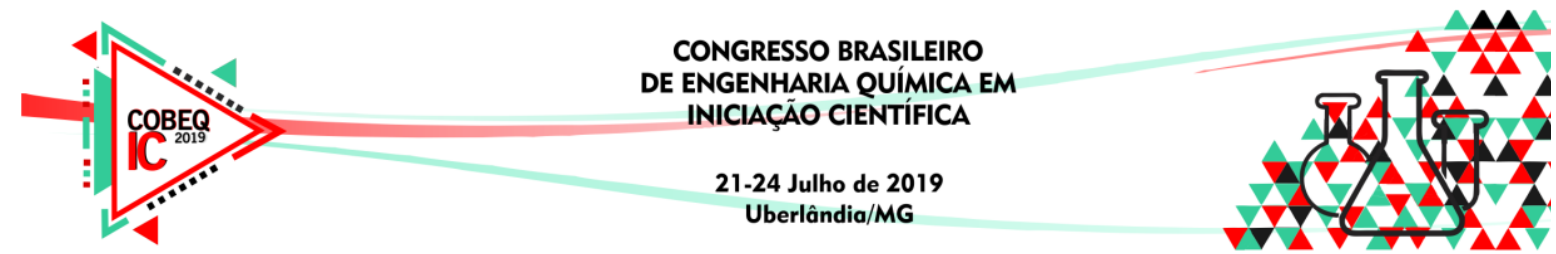

sabonetes estão de acordo com os componentes declarados nos rótulos, conforme estudo das funções desses ingredientes.

\section{CONCLUSÕES}

De acordo com a realização deste trabalho, pode-se concluir que as características dos produtos podem ser relacionadas à formulação, conforme observado por meio da compreensão da função de cada componente declarado no rótulo. Além disso, a faixa de viscosidade de um produto é estabelecida com base na forma de aplicação a ser utilizada, incluído o tipo de embalagem, e na região do corpo na qual o produto será aplicado.

O conhecimento dos produtos atualmente disponíveis e das tendências de mercado é o ponto de partida para a melhoria desses produtos e do processo produtivo, com foco na utilização de substâncias alternativas, além do uso de menor número de ingredientes na formulação. Assim, permite-se que a indústria do setor desenvolva produtos que atendam às expectativas dos consumidores, cada vez mais exigentes com relação à fabricação de produtos naturais e orgânicos, que não agridam a pele e o meio ambiente. A realização deste trabalho permitiu elaborar uma cartilha para estimular o ensino dos conceitos estudados em escolas de ensino médio, contribuindo com a popularização da ciência.

\section{REFERÊNCIAS}

ANVISA - Agência Nacional de Vigilância Sanitária. Guia de Controle de Qualidade de Produtos Cosméticos. 2 $2^{\text {a }}$ edição, revista - Brasília, 2008.

CASTRO, H.F. Sabões e Detergentes. Universidade de São Paulo: Escola de Engenharia de Lorena, $2009 . \quad$ Disponível em:< http://sistemas.eel.usp.br/docentes/arquivos/5840855/LOQ4023/apostila6Detergentes20 09[1].pdf>. Acesso em: 26 mar. 2017.

FELIPPE, C. D. S.; CRUZ, B. S.; MACEDO, B. A. B. Reologia de Fluidos Alimentícios. $16^{\circ}$ Congresso Nacional de Iniciação Científica. Universidade Santa Cecília.

FLOW, J. Cosméticos e produtos farmacêuticos. World of Rheology, 2017. Disponível em: http://www.world-of-rheology.com/pt/industrias/cosmeticosprodutos-farmaceuticos/.

Acesso em: 12 jun 2017.

ISENMANN, A. F. Princípios Químicos em Produtos Cosméticos e Sanitários. Timóteo - MG, 2015.

MENDES, B. R.; SHIMABUKURO, D. M.; UBER, M; ABAGGE, K. T. Avaliação crítica do pH dos sabonetes infantis. J. Pediatr. v.92, n.3, 2016.

NARDIN, A. D. H. G; CANDA, T. C. P. S. A.; TESCAROLLO, I. L. Produção de Sabonete Líquido de Ácido Salicílico empregando Hidroxipropil Guar como Agente de Viscosidade. Interbin, v.7 n.2, 2013. 